\title{
Ultra-Broadband Optical Transmission using Bi/Er Codoped Glass Fiber: Key Design Issue and a Survey
}

\author{
Mojaiana Synthia ${ }^{1}$ and Md. Shipon $\mathrm{Ali}^{2 *}$ \\ ${ }^{1}$ Electronics \& Communication Engineering Discipline, Khulna University, \\ Bangladesh \\ ${ }^{2}$ Senior System Engineer, Technology Division, Grameenphone Ltd., Bangladesh \\ Email*: shipon@grameenphone.com
}

\begin{abstract}
Future generation optical networks are expected to provide ultra broadband optical communication to continuously growing fiber transmission requirements. In this context, Bismuth and Erbium (Bi/Er) codoped glass fibers symbolize an important aspect as it has offered a promising active laser medium for ultra-bandwidth communication. Many studies have been demonstrated that Bi/Er codoped glass optical fiber showing ultrabroadband luminescence between 1000 and $1570 \mathrm{~nm}$ wavelength which typically covering $O, E, S, C$ and $L$ bands of optical window. But, the fluorescence profiles are highly dependent on pump wavelength. This paper provides a survey of the current researches on Bi/Er codoped glass fiber and draws their link to earlier literature on Bi-doped fiber and Er-doped fiber. Also, it present an overview on the key issues that arises during the design of a Bi/Er codoped fiber. Finally, it is intended for a wide range of readers as it covers the topics from basics to advanced aspects.
\end{abstract}

Keywords: Bismuth, Erbium, Luminescence, Laser, Bandwidth

\section{Introduction}

An increase of data traffic in telecommunication optical fiber networks demands the development of fiber lasers and wideband fiber amplifiers to cover the 1250 to1650 nm wavelength region, which is the entire optical telecommunication windows of silica fiber, for ultra-wide broadband optical communication. Optical amplifier is the key element of fiber optics communication system, continuously extending the rapid growth of wavelength division multiplexing (WDM) transmission system. The mostly used optical amplifiers are the doped fiber amplifier (DFAs) which use a doped optical fiber as a gain medium to amplify an optical signal. The signal to be amplified and a pump laser are multiplexed into the doped fiber, and the signal is amplified through interaction with the doping ions. The most common example is the Erbium Doped Fiber Amplifier (EDFA), where the core of a silica fiber is doped with trivalent erbium ions and can be efficiently pumped with a laser at a wavelength of $980 \mathrm{~nm}$ or $1480 \mathrm{~nm}$, and exhibits gain in the $1550 \mathrm{~nm}$ regions.

The introductions of water-free optical fibers have extended the accessible telecommunications windows across the entire spectrum from 1100 to $1700 \mathrm{~nm}$. It is also evident that active glass optical fibers are one of the most efficient fiber amplifiers with excellent laser media that contributes magnificent beam quality. Until recently, only rare earth-doped (especially, erbium-doped) optical fibers have been used in development of fiber lasers and optical amplifiers. At present, rare earth-doped fiber amplifiers with wavelengths in the near-IR region, 1530-1610 nm, are widely used for DWDM transmission system [1-4]. 
However, the attenuation characteristics of silica-based fibers (fig.1) demonstrate low optical losses in a considerably broader range, in particular, the spectral region from 1300 to 1700 $\mathrm{nm}$ where the optical loss is under $0.4 \mathrm{~dB} / \mathrm{km}$ and which might be used for data transmission. Hence, the efficient fiber amplifiers, key components of high-speed ultra-broadband optical fiber communication, for the spectral ranges $1300-1520 \mathrm{~nm}$ and $1610-1700 \mathrm{~nm}$ are still unavailable. However, various types of Bismuth-doped optical fibers have been studied and used to construct Bismuth-doped fiber amplifiers with gain area 1300-1500 nm [1-9]. In addition, authors of $[2,3,5,8]$ suggested that ultra broadband transmission from 1100 to $1500 \mathrm{~nm}$, and gain across $\mathrm{O}, \mathrm{E}$ and $\mathrm{S}$ bands also possible using Bi-doped silica glasses. Also, some recent results have been demonstrated potential possibility to achieve ultra-broadband gain which can possibly cover entire optical ranges from 1000 to $1700 \mathrm{~nm}$ [10-16].

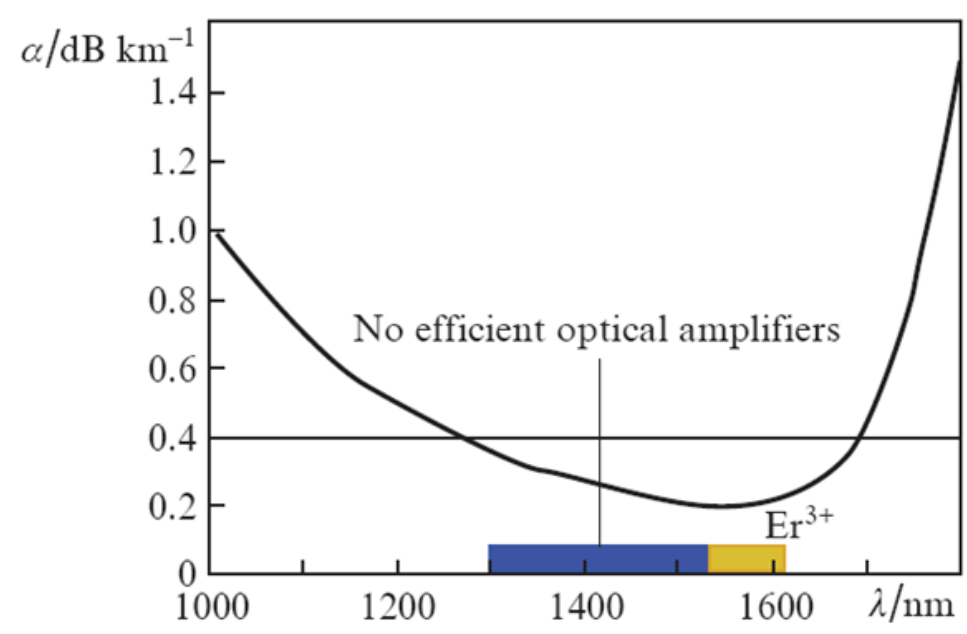

Figure 1. Attenuation Characteristics of Silica-based Fibers and the Spectral Region Where High-speed Data Transmission Currently Employed

\section{Luminescence Properties of Bi-doped Fiber}

The first bismuth-doped optical fiber was fabricated simultaneously and independently at the Fiber Optics Research Center (FORC) of Russian Academy of Sciences (RAS) in cooperation with the Institute of Chemistry of High-Purity Substances, and at Sumitomo Electric Industries Limited of Japan [2]. They fabricated the bismuth-doped fiber using modified chemical vapour deposition (MCVD) process [17], where the core consisted of bismuth-aluminium codoped silica glass that had been the first to exhibit near-IR luminescence. Figure 1 shows the luminescence spectra of the first Bi-doped optical fiber at pumping wavelength $676 \mathrm{~nm}$ [2]. Authors in [5] also provided similar luminescence spectrum of their own developed bismuth - aluminium doped silica glass which was pumped by 1064 nm laser. In addition, [9] fabricated a bismuth-doped phosphor-germanosilicate fiber in order to assess the influence of glass composition on the luminescence properties of the fibers. 


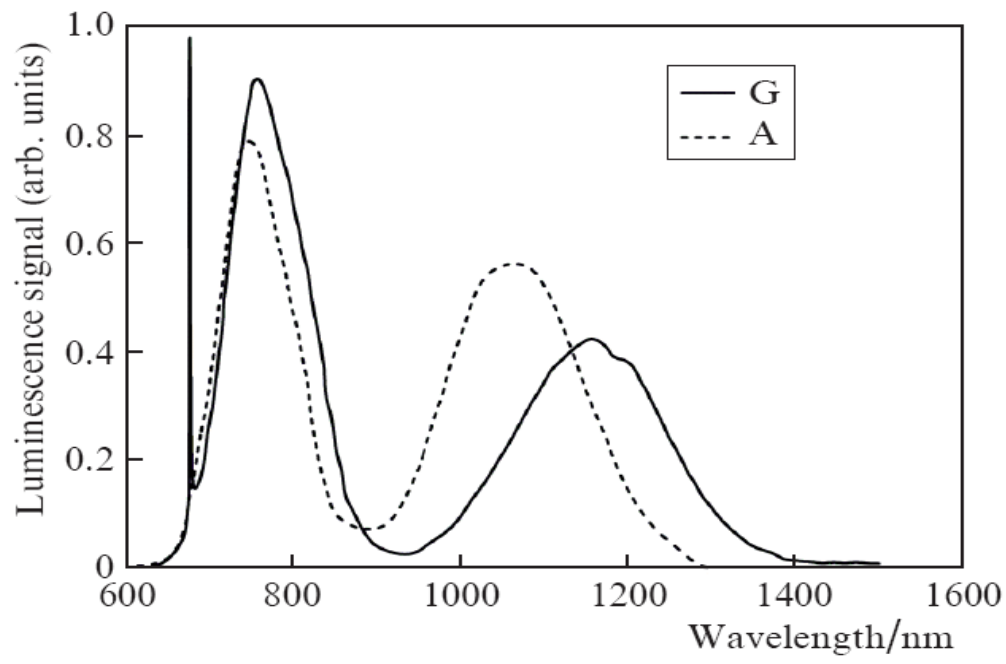

Figure 2. Luminescence Spectra of the First Produced Bi-doped Optical Fiber

Some recent studies suggested ultra broadband gain across $\mathrm{O}, \mathrm{E}$ and $\mathrm{S}$ bands, covering $1100-1500 \mathrm{~nm}$, of the telecommunications window using Bi-doped silica glasses [3, 8]. Bidoped silica fibers were also reported with luminescence in the region of 1050 to $1300 \mathrm{~nm}$ in [2]. In addition, Er-doping in Bi-based glasses [10] or in Bi-based glass fibers (Bi-EDFs) [11] has been studied to improve Er emission in $\mathrm{C}$ and $\mathrm{L}$ bands. These $\mathrm{Bi}$-doped fibers and $\mathrm{Bi}$ $\mathrm{EDFs}$ (kind of $\mathrm{Bi} / \mathrm{Er}$ codoped fibers) are developed separately for $\mathrm{O}, \mathrm{E}$ and $\mathrm{S}$ bands, and for $\mathrm{C}$ and L ands, respectively. Reference [3] suggested that Bismuth-doped silica glass (BiSG) is a new material that emits a broadband fluorescence peak at around $1250 \mathrm{~nm}$ with a bandwidth over $300 \mathrm{~nm}$.

\section{Luminescence Properties of Bi/Er Fibers}

To develop an truly ultra broadband optical fiber, gain from 1100 to $1600 \mathrm{~nm}, \mathrm{Bi} / \mathrm{Er}$ codoping in bulk glasses has been vastly targeted [10-16]. Reference [10-12] reported ultra broadband fluorescence between 1160 and $1570 \mathrm{~nm}$ using a Bi and Er codoped bulk silica glass mix melted in a crucible. Authors in [12] reported ultra broadband fluorescence between 1160 and $1580 \mathrm{~nm}$, from $\mathrm{Bi} / \mathrm{Er}$ codoped germanate glasses. Despite of these impressive achievements, no $\mathrm{Bi} / \mathrm{Er}$ codoped optical fiber has been developed with ultra broadband gain across all $\mathrm{O}, \mathrm{E}, \mathrm{S}, \mathrm{C}$ and $\mathrm{L}$ bands. Furthermore, the requirement of separate pumping of particular bands is not sufficient to confirm that true ultra broadband amplification would be possible, since it has not been confirmed that each emission band is associated with distinct defect sites. However, the codoping of the $\mathrm{Er}-\mathrm{Bi}$ ions is used to supposedly alleviate clustering and improve fluorescence efficiency for high Er concentrations is suggested in [17], although there was too little gain around $1220 \mathrm{~nm}$ and narrow emission at $1380 \mathrm{~nm}$.

In 2012, authors in [13] acknowledged ultra broadband optical fiber with luminescent over 1100-1570 nm using $\mathrm{Bi} / \mathrm{Er} / \mathrm{Al} / \mathrm{P}$ codoped germane-silica fiber. Also, they reported on simultaneous pumping of the fiber to confirm that the ultra broadband fluorescence obtained by selective pumping is in fact of additive which suggests true simultaneous and ultra broadband emission is feasible. The process has involved fabricating fiber by in-situ modified chemical vapor deposition (MCVD) doping [17]. The fiber was pumped by $532 \mathrm{~nm}$ and 808 nm pumping laser. The results depicted an excellent luminescence intensity index over 1100- 
$1570 \mathrm{~nm}$ [13], which expedite the possibilities of development of ultra broadband fiber amplifiers in telecommunications.

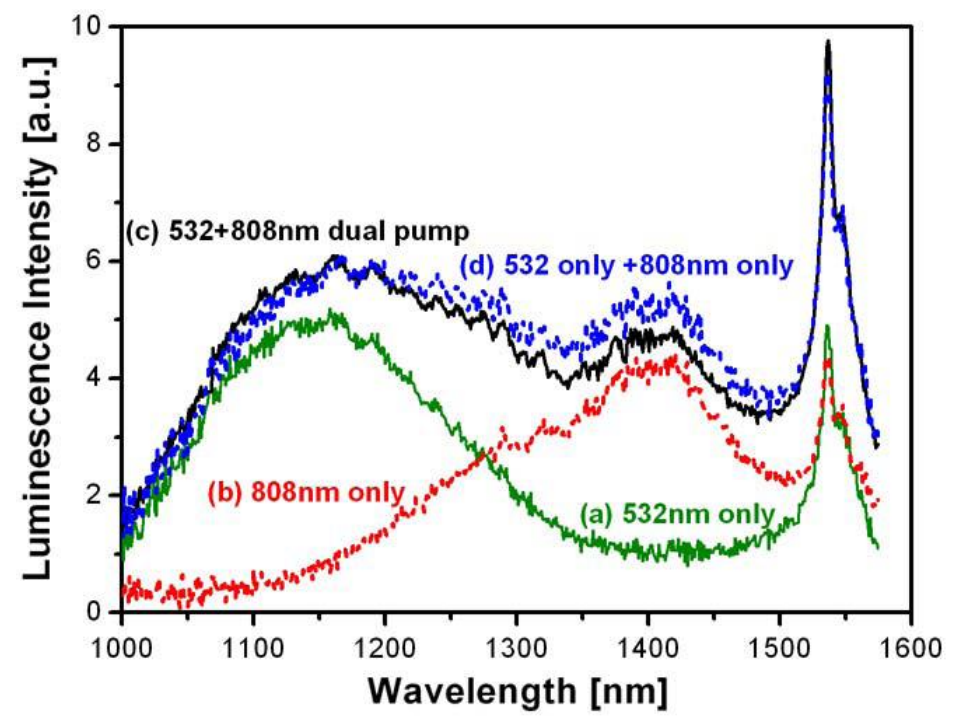

\section{Figure 3. Luminescence of the Bi/Er Codoped Fiber Pumped by Various Wavelength Lasers}

The fluorescence of the $\mathrm{Bi} / \mathrm{Er}$ codoped fiber, pumped by $532 \mathrm{~nm}$ and $808 \mathrm{~nm}$ laser, is shown in Figure 3 [13]. It is seen that there are two Bi related defect sites and one Er defect site. The existence of two separate bands suggests that due to the glassy nature of the $\mathrm{Bi}$ incorporation and its place on the periodic table, there at least two types of Bismuth defects exist in Bi/Er codoped multi-component glass core fiber. In addition, the location and FWHM of the emission peak of the Bismuth center depends significantly upon the pump wavelengths and its physical environment [13]. Whereas, the location and FWHM of the emission peak of Erbium is an almost unchanged regardless wavelength. However, spectral additivity that obtained by using two co-propagating pumps $(532$ and $808 \mathrm{~nm})$ to produce ultrabroadband luminescence is also visualized in Figure 3. It is clear that the fluorescence using two pumps is nearly the same as the linear addition of the individual fluorescence of the two pumps. The FWHM from two pumps is about $450 \mathrm{~nm}$ from 1050 to $1500 \mathrm{~nm}$, and the product of stimulated emission cross section and emission lifetime was theoretically estimated to be about $1.8 \times 10^{-24} \mathrm{~cm}^{2}$, showing promising potential as an ultra-broadband gain medium [13, $16,20]$.

\section{Conclusions}

In this paper, we have described a brief historical development of optical fiber communication system in terms of bandwidth expansion. Here, the most recent glass fiber is elaborately discussed, and the challenge of utilization is also mentioned. The results obtained so far confirm that $\mathrm{Bi} / \mathrm{Er}$ codoped glass fibers have great potential as near-IR lasing media. The luminescence spectra of various $\mathrm{Bi} / \mathrm{Er}$ codoped glasses cover the entire spectral range $800-2000 \mathrm{~nm}$ and have luminescence bands $300-450 \mathrm{~nm}$ in width. However, the gain band is not equalized throughout the luminescence band. In addition, the exact nature of near-IR emitting bismuth centers is still unclear and efficient operation of bismuth-doped fiber lasers is achieved only at very low bismuth concentrations which also have some severe drawbacks. 
It may be that these drawbacks will be eliminated when the nature of the bismuth-related active centers in glasses will be better understood.

\section{References}

[1] E. M. Dianov, V. V. Dvoyrin, V. M. Mashinsky, A. A. Umnikov, M. V. Yashkov and A. N. Guranov, "CW bismuth Laser", Quantum Electronics, vol. 35, (2005), pp. 1083-1084.

[2] E. M. Dianov, V. V. Dvoyrin, V. M. Mashinsky, A. A. Umnikov, M. V. Yashkov and A. N. Guranov, "Bidoped Silica Fibers - a New Active Medium for Tunable Fiber Lasers and Broadband Fiber Amplifiers", Opt. Lett., vol. 31, no. 20, (2006), pp. 2966-2968.

[3] Young-Seok Seo and Yasushi Fujimoto, "Bismuth-doped Silica Fiber Amplifier", InTech, (2010).

[4] V. V. Dvoyrin, V. M. Mashinsky and E. M. Dianov, "Yb-Bi Pulsed Fiber Lasers", Opt. Lett.,vol. 32, no. 5, (2007), pp. 451-453.

[5] Y. Fujimoto \& M. Nakatsuka, "Infrared Luminescence from Bismuth-doped Silica Glass", Jpn. J. Appl. Phys., vol. 40, no. 3B, (2001), pp. 279-281.

[6] Y. Fujimoto and M. Nakatsuka, "Optical Amplification in Bismuth-doped Silica Glass", Appl. Phys. Lett., vol. 82 no. 19, (2003), pp. 3325-3326.

[7] Bufetov and E. M. Dianov, "Bi-doped Fiber Lasers", Laser Phys. Lett., vol.6, no.7, (2009), pp. 487-504.

[8] M. Melkumov, I. Bufetov, A. Shubin, S. Firstov \& V. Khopin, "LD Pumped Bismuth-doped Optical Fiber Amplifier for $1430 \mathrm{~nm}$ Band", Opt. Lett., vol. 36, (2011), pp. 2408-2410.

[9] T. Ohkura, Y. Fujimoto, M. Nakatsuka and Y. Seo, "Local Structures of Bismuth Ion in Bismuth-doped Silica Glasses Analyzed Using Bi LIII X-ray Absorption Fine Structure”, J. Am. Ceram. Soc., vol. 90, no. 11, (2007), pp. 3596-3600.

[10] H. Y. Tam, W. H. Chung, B. O. Guan, H. L. Liu, P. K. A. Wai, and N. Sugimoto, "Development of $\mathrm{Bi}_{2} \mathrm{O}_{3}-$ based Erbium-doped Fibers", Proc. SPIE, vol. 5644, (2005), pp. 259-264.

[11] M. Peng, N. Zhang, L. Wondraczek, J. Qiu, Z. Yang, and Q. Zhang, "Ultrabroad NIR luminescence and Energy Transfer in Bi and Er/Bi co-doped Germanate Glasses", Opt. Express, vol.19, (2011), pp. 20799.

[12] Y. Kuwada, Y Fujimoto, and M.Nakatsuka, "Ultra-wideband Light Emission from Bismuth and Erbium Doped Silica” Jpn. J. Appl. Phys., vol. 46, (2007), pp. 1531-1532.

[13] Y. Luo, J. Wen, J. Zhang, J. Canning and G. D. Peng, "Bismuth and Erbium Codoped Optical Fiber with Ultra Broadband Luminescence Across O-, E-, S-, C-, and L-bands”, Opt. Lett., vol. 37, no. 16, (2012), pp. 3447-3449.

[14] M. Peng, J. Qiu, D. Chen, X. Meng and C. Zhu, "Broadband Infrared Luminescence from $\mathrm{Li}_{2} \mathrm{O}-\mathrm{Al}_{2} \mathrm{O}_{3}-\mathrm{ZnO}$ $\mathrm{SiO}_{2}$ Glasses Doped with $\mathrm{Bi}_{2} \mathrm{O}_{3}$ ”, Opt. Express, vol. 13, no. 18, (2005), pp.6892-6898.

[15] M. Peng, J. Qiu, D. Chen, X. Meng and C. Zhu, "Investigations on Bismuth and Aluminum Co-doped Germanium Oxide Glasses for Ultra-broadband Optical Amplification”, J. Non-Cryst. Solids, vol. 351, (2005), pp. 2388-2393.

[16] Y. Q. Qiu, X. Y. Dong, and C. L. Zhao, "Spectral Characteristics of the Erbium-bismuth Co-doped Silica Fibers and its Application in Single Frequency Fiber Laser", Laser Phys. Lett., vol. 20, (2010), pp. 1418 1423.

[17] A. S. Webb, A. J. Boyland, R. J. Standish, S. Yoo, J. K. Sahu, and D. N. Payne, "MCVD in-situ Solution Doping Process for the Fabrication of Complex Design Large Core Rare-earth Doped Fibers", J. Non-Cryst. Solids, vol. 356, (2010), pp. 848-852.

[18] Y. Seo, Y. Fujimoto and M. Nakatsuka, "Amplification in a Bismuth-doped Silica Glass at Second Telecommunication Windows", Conference on Lasers and Electro-Optics, CThR6, (2005) May.

[19] P. C. Becker, N. A. Olsson, and J. R. Simpson, "Erbium-doped Fiber Amplifiers Fundamentals and Technology", Academic publications, chap.1 and 4, (1999).

[20] A. Tunnermann, T. Schreiber, and J. Limpert, "Fiber Lasers and Amplifiers: An Ultrafast Performance Evolution”, Appl. Opt., vol. 49, no. 25, (2010), pp. 71-78. 


\section{Authors}

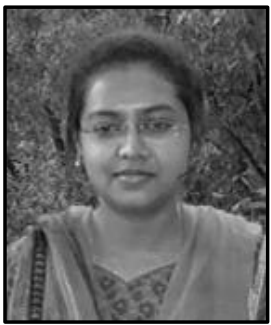

Mojaiana Synthia, has received B.Sc. in Electronics and Communication Engineering degree from Khulna University, Bangladesh in 2009. She worked in a telecom industry for a short tenure. Currently she is working in different research projects related to communication system. Her research interests are Optical communication, Cognitive radio network and 4G mobile communication. Now, she is looking toward in her $\mathrm{PhD}$ degree.

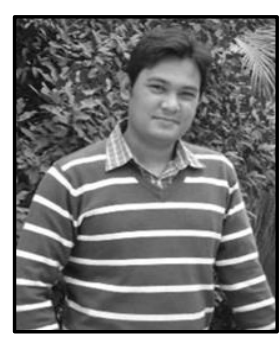

Md. Shipon Ali, achieved his B.Sc. in Electronics \& Communication Engineering degree from Khulna University, Bangladesh in 2009. He achieved 'University Gold Medal' from Khulna University for securing first position with distinction by obtaining the highest CGPA in Bachelor Examinations among all disciplines under School of Science, Engineering and Technology in the session 2007-2008. Now, he is working as a 'senior Engineer' in a leading telecom service provider in Bangladesh. His research interests are optical communication system, $4 \mathrm{G}$ mobile communications and digital signal processing system. 